Bull. Austral. Math. Soc.

20F16, 20E25

VOL. $61(2000) \quad[33-38]$

\title{
CHARACTERISATION OF NILPOTENT-BY-FINITE GROUPS
}

\section{NADIR TRABELSI}

Let $G$ be a finitely generated soluble group. The main result of this note is to prove that $G$ is nilpotent-by-finite if, and only if, for every pair $X, Y$ of infinite subsets of $G$, there exist an $x$ in $X, y$ in $Y$ and two positive integers $m=m(x, y)$, $n=n(x, y)$ satisfying $\left[x, n y^{m}\right]=1$. We prove also that if $G$ is infinite and if $m$ is a positive integer, then $G$ is nilpotent-by-(finite of exponent dividing $m$ ) if, and only if, for every pair $X, Y$ of infinite subsets of $G$, there exist an $x$ in $X, y$ in $Y$ and a positive integer $n=n(x, y)$ satisfying $\left[x, n y^{m}\right]=1$.

\section{INTRODUCTION AND RESULTS}

Following questions of Erdös, B.H. Neumann proved in [9] that a group is centreby-finite if, and only if, every infinite subset contains a commuting pair of distinct elements. From this, as was observed in [7], it is easy to show that if $G$ is an infinite group such that for every pair $X, Y$ of infinite subsets of $G$, there exist an $x$ in $X$ and $y$ in $Y$ that commute, then $G$ is Abelian. Endimioni [2, Theorem 2] extended this result, by proving that if $G$ is an infinite finitely generated soluble group such that for every pair $X, Y$ of infinite subsets of $G$, there exist an $x$ in $X, y$ in $Y$ and a positive integer $n=n(x, y)$ satisfying $\left[x,{ }_{n} y\right]=1$, then $G$ is nilpotent. The main purpose of this note is to improve this last result. We shall prove:

THEOREM 1. Let $G$ be a finitely generated soluble group. Then the following properties are equivalent:

(i) $G$ is nilpotent-by-finite.

(ii) For every pair $X, Y$ of infinite subsets of $G$, there exist an $x$ in $X$, $y$ in $Y$ and two positive integers $m=m(x, y), n=n(x, y)$ satisfying $\left[x,{ }_{n} y^{m}\right]=1$.

From a result of Lennox [4], a finitely generated soluble group all of whose twogenerator subgroups are nilpotent-by-finite, is itself nilpotent-by-finite. As an immediate consequence of Theorem 1, we have the following generalisation of Lennos's result:

\section{Received 1999}

This work was achieved during a visit at the 'Centre de Mathématiques et d'Informatique' of the University of Marseilles. I would like to thank Dr. G. Endimioni for his suggestions and his hospitality.

Copyright Clearance Centre, Inc. Serial-fee code: 0004-9727/00 \$A2.00+0.00. 
COROLlary 1. A finitely generated soluble group $G$ is nilpotent-by-finite if, and only if, for every pair $X, Y$ of infinite subsets of $G$, there exist an $x$ in $X$ and $y$ in $Y$ generating a nilpotent-by-finite group.

As a consequence of Theorem 1 , we shall prove the result:

COROLlaRY 2. Let $G$ be a finitely generated soluble group such that for every pair $X, Y$ of infinite subsets of $G$, there exist an $x$ in $X, y$ in $Y$ and a positive integer $m=m(x, y)$ satisfying $\left[x, y^{m}\right]=1$. Then $G$ is Abelian-by-finite.

Corollary 2 leads us to consider the following question: if the integers $n(x, y)$ of Theorem 1 are bounded by an integer $k$, then is $G$ a finite extension of a nilpotent group of class at most an integer depending only on $k$ ? We are unable to answer this in the general case. However, we shall prove:

THEOREM 2. Let $G$ be a finitely generated metabelian group satisfying the condition (ii) of Theorem 1, and suppose that the integers $n(x, y)$ are bounded by a positive integer $k$. Then there is a function $c(k)$ of $k$ only, such that $G$ is a finite extension of a nilpotent group of class at most $c(k)$.

Note that these results are not true for arbitrary groups. Indeed, Golod [3] showed that for each integer $d>1$ and each prime $p$, there are infinte $d$-generator groups all of whose $(d-1)$-generator subgroups are finite $p$-groups. For $d=3$, we obtain groups which satisfy the combinatorial conditions of the theorems and the corollaries, but which are not nilpotent-by-finite.

Now we turn our attention to the integers $m(x, y)$. We shall prove:

TheOREM 3. Let $m$ be a positive integer and let $G$ be an infinite finitely generated soluble group. Then the following properties are equivalent:

(i) $G$ is nilpotent-by-(finite of exponent dividing $m$ ).

(ii) For every pair $X, Y$ of infinite subsets of $G$, there exist an $x$ in $X, y$ in $Y$ and a positive integer $n=n(x, y)$ such that $\left[x,{ }_{n} y^{m}\right]=1$.

If we take $m=1$, then we find again [2, Theorem 2].

Our notation and terminology are the usual ones, and can be found in [10]. In particular, $[x, n y]$ is defined for each integer $n \geqslant 0$ by $[x, 0 y]=x$ and $\left[x,_{n+1} y\right]=$ $\left[\left[x,{ }_{n} y\right], y\right]$. We shall denote by $\Omega^{*}$ the class of groups satisfying the condition (ii) of Theorem 1.

\section{Some PRELIMINARY LEMmas}

LEMma 1. Let $G$ be a finitely generated metabelian group in the class $\Omega^{*}$. Then $G$ is nilpotent-by-finite. 
PROOF: Let $G$ be a finitely generated metabelian group in the class $\Omega^{*}$. Suppose that $G$ is not nilpotent-by-finite. Since $\Omega^{*}$ is a quotient closed class of groups, and since finitely generated nilpotent-by-finite groups are finitely presented, it follows, by [10, Lemma 6.17], that we may assume that every proper homomorphic image of $G$ is nilpotent-by-finite. Since $G$ is metabelian, its Hirsch-Plotkin radical $H$ is non trivial; hence, $G / H$ is nilpotent-by-finite. It follows that $G$ contains a normal subgroup $K$ of finite index such that $K / H$ is nilpotent. If $K / H$ is infinite, then it contains an element $y H$ of infinite order [10, Theorem 2.24]. Thus, for any integer $k, y^{k} \notin H$; furthermore, for any $x \in G$, the subsets $\left\{y^{i} x: i\right.$ positive integer $\}$ and $\left\{y^{i}: i\right.$ positive integer $\}$ are infinite. Hence, there exist positive integers $r, k, m=m(x, y)$ and $n=n(x, y)$ such that $\left[y^{r} x,_{n} y^{k m}\right]=1$; so we get that $\left[x,_{n} y^{k m}\right]=1$. Since $G$ is a finitely generated metabelian group, it is eremitic [5, Theorem B]. This means that there is a positive integer $d$, depending only on $G$, such that $\left[a, b^{d}\right]=1$ whenever $\left[a, b^{i}\right]=1$, for any $a, b$ in $G$ and any positive integer $i$. Therefore, we deduce that $\left[\left[x,{ }_{n-1} y^{k m}\right], y^{d}\right]=1$ : The group $G$ being metabelian, it is easy to see that $[a, b, c]=[a, c, b]$ for all elements $a, b, c$ of $G$ such that $b c=c b$. Thus, we get that $\left[\left[x, y^{d}\right]_{n-1} y^{k m}\right]=1$; and by induction on $n$, we obtain that $\left[x,{ }_{n} y^{d}\right]=1$. Therefore, $y^{d}$ is a left Engel element of $G$. Since $G$ is metabelian, the set of left Engel elements of $G$ coincides with its Hirsch-Plotkin radical [10, Theorem 7.34]. So $y^{d} \in H$, and this contradicts the choice of $y$. It follows that $K / H$ is finite, so $G / H$ is finite. Since $G$ is finitely generated, $H$ is also finitely generated. Hence, $H$ is nilpotent; and $G$ is, therefore, nilpotent-by-finite, a contradiction which completes the proof.

We shall use the following lemma which is due to Lennox [6].

LEMMA 2. Let $G$ be a finitely generated soluble group and $A$ a normal Abelian subgroup such that $G / A$ is polycyclic and $\langle a, g\rangle$ is polycyclic whenever $a \in A$ and $g \in G$. Then $G$ is polycyclic.

LEMmA 3. Let $G$ be a finitely generated soluble group in $\Omega^{*}$. Then $G$ is polycyclic.

Proof: Since polycyclic groups are finitely presented, and since $\Omega^{*}$ is a quotient closed class of groups, by [10, Lemma 6.17], we may assume that every proper homomorphic image of $G$ is polycyclic, but $G$ itself is not polycyclic. Since $G$ is soluble, it has a non trivial normal Abelian subgroup $A$; so $G / A$ is polycyclic. Let $g \in G$ and $a \in A ;\langle a, g\rangle$ is, therefore, a finitely generated metabelian group in the class $\Omega^{*}$. It follows, from Lemma 1 , that $\langle a, g\rangle$ is nilpotent-by-finite. Thus, $\langle a, g\rangle$ is polycyclic. From Lemma 2, we can deduce that $G$ is polycyclic, which is a contradiction.

\section{Proofs of the Results}

Proof of Theorem 1: Clearly we have only to show that (ii) implies (i). Suppose 
that $G$ is a finitely generated soluble group in the class $\Omega^{*}$; from Lemma $3, G$ is polycyclic. The group $G$ contains, therefore, a normal subgroup $H$ of finite index, whose derived subgroup $H^{\prime}$ is nilpotent $[11,15.1 .6]$. Since $G$ is polycyclic, it satisfies the maximal condition on normal subgroups; and since $\Omega^{*}$ is a quotient closed class, we may, therefore, assume that $G$ is not nilpotent-by-finite, but that every proper homomorphic image of $G$ is nilpotent-by-finite. If $H^{(2)}$, the third term of the derived series of $H$, is non trivial, then $G / H^{(2)}$ is nilpotent-by-finite. Hence, $G$ contains a normal subgroup $K$ of finite index such that $K / H^{(2)}$ is nilpotent. Now $K / H^{(2)}$ and $H^{\prime} / H^{(2)}$ are two normal nilpotent subgroups of $G / H^{(2)}$, so their product $K H^{\prime} / H^{(2)}$ is nilpotent [10, Theorem 2.18]. Also $H^{\prime}$ and $K H^{\prime} / H^{(2)}$ are nilpotent; by a result of Hall [10, Theorem 2.27], $K H^{\prime}$, and so $K$, is nilpotent. Thus, $G$ is nilpotent-byfinite, which is a contradiction. So $H^{(2)}=1$ and $H$ is, therefore, a finitely generated metabelian group. It follows, from Lemma 1 , that $H$ is nilpotent-by-finite. So $G$ is nilpotent-by-finite, a contradiction which completes the proof.

Proof of Corollary 2: Let $G$ be a finitely generated soluble group such that, for every pair $X, Y$ of infinite subsets of $G$, there exist an $x$ in $X, y$ in $Y$ and a positive integer $m=m(x, y)$ satisfying $\left[x, y^{m}\right]=1$. Clearly, we may assume that $G$ is infinite. It follows, from Theorem 1 , that $G$ is nilpotent-by-finite. Thus, $G$ has an infinite finitely generated nilpotent subgroup of finite index so, without loss of generality, we may suppose $G$ is finitely generated and nilpotent. Since finitely generated nilpotent groups are (torsion-free)-by-finite $[11,5.4 .15(\mathrm{i})]$, we may assume also that $G$ is torsionfree. The group $G$, being nilpotent and finitely generated, contains a maximal normal Abelian subgroup $A$. We know that $C_{G}(A)=A[11,5.2 .3]$. Let $a$ be a non trivial element of $A$, and let $g \in G$; since $G$ is torsion-free, the subsets $\left\{a^{i}: i\right.$ integer $\}$ and $\left\{a^{i} g: i\right.$ integer $\}$ are infinite. There exist, therefore, integers $i, j$ and $m=m(a, g)$ such that $\left[a^{i},\left(a^{j} g\right)^{m}\right]=1$. Since $A$ is a normal Abelian subgroup of $G$, we get that $\left[a,\left(a^{j} g\right)^{m}\right]^{i}=1$. Thus, we obtain that $\left[a,\left(a^{j} g\right)^{m}\right]=1$, because $G$ is torsionfree; hence, it is easy to deduce that $\left[a, g^{m}\right]=1$. The group $G$, being nilpotent and finitely generated, is eremitic [5, Theorem B]. There is, therefore, a positive integer $d$, depending only on $G$, such that $\left[a, g^{d}\right]=1$; so $g^{d} \in C_{G}(A)$. Now $A=C_{G}(A)$, thus $g^{d} \in A$. It follows, that $G / A$ is a periodic group. Therefore, $G / A$ being a periodic finitely generated nilpotent group, is finite. Hence, $G$ is Abelian-by-finite, as required.

PROOF OF THEOREM 2: Let $G$ be a finitely generated metabelian group in the class $\Omega^{*}$, such that the integers $n(x, y)$ are bounded by a positive integer $k$. Clearly, we may assume that $G$ is infinite. It follows, from Theorem 1 , that $G$ is nilpotentby-finite. Hence, $G$ contains a normal nilpotent subgroup $H$ of finite index. Since finitely generated nilpotent groups are (torsion-free)-by-finite $[11,5.4 .15$ (i)], there is 
no loss of generality if we assume that $H$ is torsion-free. Since $G$ is infinite, $H$ is an infinite finitely generated nilpotent group. Hence, $\zeta(H)$, the centre of $H$, is infinite [10, Theorem 2.24]. Thus, for any $x, y$ in $H$, the subsets $x \zeta(H)$ and $y \zeta(H)$ are infinite. There exist, therefore, $a, b$ in $\zeta(H)$ and integers $n=n(x, y), m=m(x, y)$ such that $\left[x a,{ }_{n}(y b)^{m}\right]=1$; so $\left[x,{ }_{n} y^{m}\right]=1$. Now $n \leqslant k$, so $\left[x, k y^{m}\right]=1$. Since $G$ is a finitely generated metabelian group, it is eremitic [5, Theorem B]. We proceed then as in Lemma 1 ; there is, therefore, a positive integer $d$, depending only on $H$, such that for any $x, y$ in $H$, we have $\left[x,{ }_{k} y^{d}\right]=1$. So $y^{d}$ is a left $k$-Engel element of $H$. Since $H$ is a finitely generated nilpotent group, then, according to a result of Mal'cev [8], the set $\left\{h^{d}: h \in H\right\}$ contains a normal subgroup $K$ of $H$, of finite index in $H$. Since for any $x, y$ in $H$ we have $\left[x^{d},{ }_{k} y^{d}\right]=1$ then $K$ is a $k$-Engel group. By a result of Zelmanov [12], there is an integer $c=c(k)$, depending only on $k$, such that $K$ is nilpotent of class at most $c(k)$. Hence, $H$, and therefore $G$, is a finite extension of a nilpotent group of class at most $c(k)$ as required.

Proof of Theorem 3: Clearly, every nilpotent-by-(finite of exponent dividing $m$ ) group satisfies the condition (ii). Now suppose that $G$ is an infinite finitely generated soluble group in the class $\Omega^{*}$, such that the integer $m$ is the same for any pair of infinite subsets $X, Y$ of $G$. We have to show that $G$ is an extension of a nilpotent group by a finite group of exponent dividing $m$. Since $G$ is a finitely generated soluble group, $G / G^{m}$ is a finite group of exponent dividing $m[11,5.4 .11]$. It suffices, therefore, to show that $G^{m}$ is nilpotent; and from a result of Robinson and Wehrfritz [11, 15.5.3], it suffices to show that any finite homomorphic image of $G^{m}$ is nilpotent. Let $N$ be a normal subgroup of $G^{m}$, of finite index. Since $G / G^{m}$ is finite, $N$ is of finite index in $G$. Hence, there is a $G$-admissible subgroup $M$ of $N$, of finite index in $G$. So, if $T$ is a left transversal of $M$ in $G$, then $T$ is finite; and since $G$ is infinite, $M$ is also infinite. Thus, for any $x, y$ in $T$, the subsets $x M$ and $y M$ are infinite. There exist, therefore, $a, b$ in $M$ and an integer $n=n(x, y, M)$, such that $\left[x a,{ }_{n}(y b)^{m}\right]=1 ;$ so $\left[x,_{n} y^{m}\right] \in M$. Since $T$ is finite, it follows that there is a positive integer $n$, depending only on $M$, such that for any $x, y$ in $T$, we have $\left[x, n y^{m}\right] \in M$. This means that $G / M$ satisfies the identity $\left[x,_{n} y^{m}\right]=1$, and from the corollary of $[1],(G / M)^{m}$ is, therefore, nilpotent. Now $(G / M)^{m}=G^{m} / M$, so $G^{m} / M$ is nilpotent. Hence, $G^{m} / N$, as a homomorphic image of a nilpotent group, is nilpotent.

\section{REFERENCES}

[1] O. Chapuis, 'Variétés de groupes et $m$-identities', C.R. Acad. Sci. Paris Ser, I 316 (1993), 15-17.

[2] G. Endimioni, 'Groups covered by finitely many nilpotent subgroups', Bull. Austral. Math. Soc. 50 (1994), 459-464. 
[3] E.S. Golod, 'Some problems of Burnside type', Amer. Math. Soc. Thansl. Ser. 284 (1969), 83-88.

[4] J.C. Lennox, 'Bigenetic properties of finitely generated hyper-(abelian-by-finite) groups', J. Austral. Math. Soc. 16 (1973), 309-315.

[5] J.C. Lennox and J.E. Roseblade, 'Centrality in finitely generated soluble groups', J. Algebra 16 (1970), 399-435.

[6] J.C. Lennox and J. Wiegold, 'Extensions of a problem of Paul Erdös on groups', J. Austral. Math. Soc. Ser. A 31 (1981), 459-463.

[7] P. Longobardi, M. Maj and A.H. Rhemtulla, 'Infinite groups in a given variety and Ramsey's theorem', Comm. in Algebra 20 (1992), 127-139.

[8] A. I. Mal'cev, Collected works (Nauka, Moscow, 1976).

[9] B.H. Neumann, 'A problem of Paul Erdös on groups', J. Austral. Math. Soc. Ser. A 21 (1976), 467-472.

[10] D.J.S. Robinson, Finiteness conditions and generalized soluble groups (Springer-Verlag, Berlin, Heidelberg, New York, 1972).

[11] D.J.S. Robinson, A course in the theory of groups (Springer-Verlag, Berlin, Heidelberg, New York, 1982).

[12] E.I. Zelmanov, 'On some problems of group theory and Lie algebras', Math. USSR-Sb 66 (1990), 159-168.

\footnotetext{
Département de Mathématiques

Université Ferhat Abbas

Sétif 19000

Algérie

e-mail maths@elhidhab.cerist.dz
} 